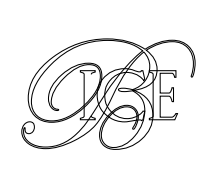

\title{
LA ESTRATEGIA DE INTERNACIONALIZACIÓN DE LA ECONOMÍA ESPAÑOLA 2017-2027
}

El Consejo de Ministros aprobó, por el Acuerdo de 8 de septiembre de 2017, la Estrategia de Internacionalización de la Economía Española 2017-2027 y el primer Plan de Acción Bienal (2017-2018) incluido en la misma. Este documento ha sido elaborado por el Ministerio de Economía, Industria y Competitividad a través de la Secretaría de Estado de Comercio, con la participación de todos los ministerios con actividad en materia de internacionalización, consultadas las comunidades autónomas y el sector privado. La estrategia y los planes de acción bienales tienen como objetivo general afianzar la contribución positiva y permanente del sector exterior a nuestro crecimiento económico, consiguiendo así una mayor creación de empleo.

Palabras clave: comercio exterior, política comercial, política de internacionalización.

Clasificación JEL: F13, F14.

\section{Introducción}

El Consejo de Ministros del 8 de septiembre de 2017 aprobó la Estrategia de Internacionalización de la Economía Española 2017-2027 y el primer Plan de Acción Bienal (2017-18) incluido en la misma. En cumplimiento de lo establecido en la Ley $14 / 2013$, de 27 de septiembre, de apoyo a los emprendedores y su internacionalización, ambos han sido elaborados por el Ministerio de Economía, Industria y Competitividad a través de la Secretaría de Estado de Comercio, con la participación de

*Este artículo ha sido redactado por Carmen Laín Valenzuela y Ana Robles González, Subdirectora General y Subdirectora General Adjunta de Análisis y Estrategia de Internacionalización, respectivamente. Dirección General de Política Comercial y Competitividad. Secretaría de Estado de Comercio.

Versión de octubre de 2017. todos los ministerios con actividad en materia de internacionalización, consultadas las comunidades autónomas y el sector privado.

Desde el año 2014, el superávit por cuenta corriente de nuestra economía ha venido acompañado de cifras positivas de crecimiento del PIB, algo que no sucedía desde hace más de 25 años. Con el fin de asegurar que la contribución positiva del sector exterior al crecimiento económico se convierta en un fenómeno estructural y revierta en la creación de empleo, es necesario contar con una estrategia a medio y largo plazo que permita la adecuada articulación de los mecanismos que impulsan la internacionalización de la economía española.

La Ley 14/2013, de 27 de septiembre, de apoyo a los emprendedores y su internacionalización, $D$ 
contempla un concepto amplio de internacionalización, al establecer que las políticas de fomento de la misma son el conjunto de las actuaciones que desarrolla el sector público, junto con el sector privado, para facilitar y reforzar la dimensión internacional de la economía española y fomentar la presencia exterior de las empresas y de los emprendedores como factores de estabilidad, crecimiento y generación de empleo.

En el marco de esta ley se aprobó el Plan Estratégico de Internacionalización de la Economía Española 2014-2015, que supuso un esfuerzo notable por coordinar a todos los actores implicados en la internacionalización, siendo el Ministerio de Economía, Industria y Competitividad el competente para realizar dicha coordinación. Finalizado el periodo del Gobierno en funciones, el objetivo de la Secretaría de Estado de Comercio ha sido contar con una estrategia en materia de internacionalización que sea una política de Estado, tal como reclama el sector privado, independiente de la orientación política del Gobierno. Si bien la ley mencionada establecía el carácter bienal de los planes estratégicos de internacionalización, la experiencia en la elaboración, ejecución y evaluación del primer plan estratégico ha puesto de manifiesto la necesidad de contar con un marco que permita una planificación a más largo plazo.

De ahí la elaboración de una estrategia a diez años, que se irá desarrollando mediante planes de acción bienales adaptados a las circunstancias que impacten en el comercio mundial en cada momento. La Estrategia de Internacionalización 2017-2027 y el Plan de Acción 2017-2018, aprobados por el Consejo de Ministros el 8 de septiembre, son el fruto del trabajo del Grupo de Trabajo Interministerial de Apoyo a la Internacionalización de la empresa española. En su elaboración han participado, en una primera fase, los centros directivos del
Ministerio de Economía, Industria y Competitividad, en particular la Dirección General de Comercio Internacional e Inversiones, ICEX España Exportación e Inversiones y los instrumentos financieros de apoyo a la internacionalización FIEM (Fondo de Internacionalización de la Empresa), COFIDES (Compañía Española de Financiación del Desarrollo), CESCE (Compañía Española de Seguro de Crédito a la Exportación) e ICO (Instituto de Crédito Oficial). Tanto el sector privado - representado principalmente a través de las organizaciones empresariales, cámaras de comercio y las principales asociaciones de exportadores- como las comunidades autónomas han sido consultados en el proceso.

La estrategia comienza identificando las fortalezas y debilidades de la economía española en el ámbito de la internacionalización en el momento actual y, a partir de ahí, define los objetivos y principales ejes de actuación para maximizar la contribución del sector exterior al crecimiento y a la creación de empleo. Incorpora, asimismo, la definición de un conjunto de indicadores, asociados a los objetivos, que permita realizar un seguimiento pormenorizado del cumplimiento de las metas fijadas en la estrategia y en los planes que la desarrollan, así como de su grado de eficiencia y efectividad. Sobre esa base, se implementarán procesos de evaluación de impacto y resultados, que será cada cinco años en el caso de la estrategia y con periodicidad bienal en el caso de los planes.

\section{Cambios recientes experimentados por el sector exterior español}

En los últimos ocho años, el sector exterior español ha experimentado cambios considerables. 

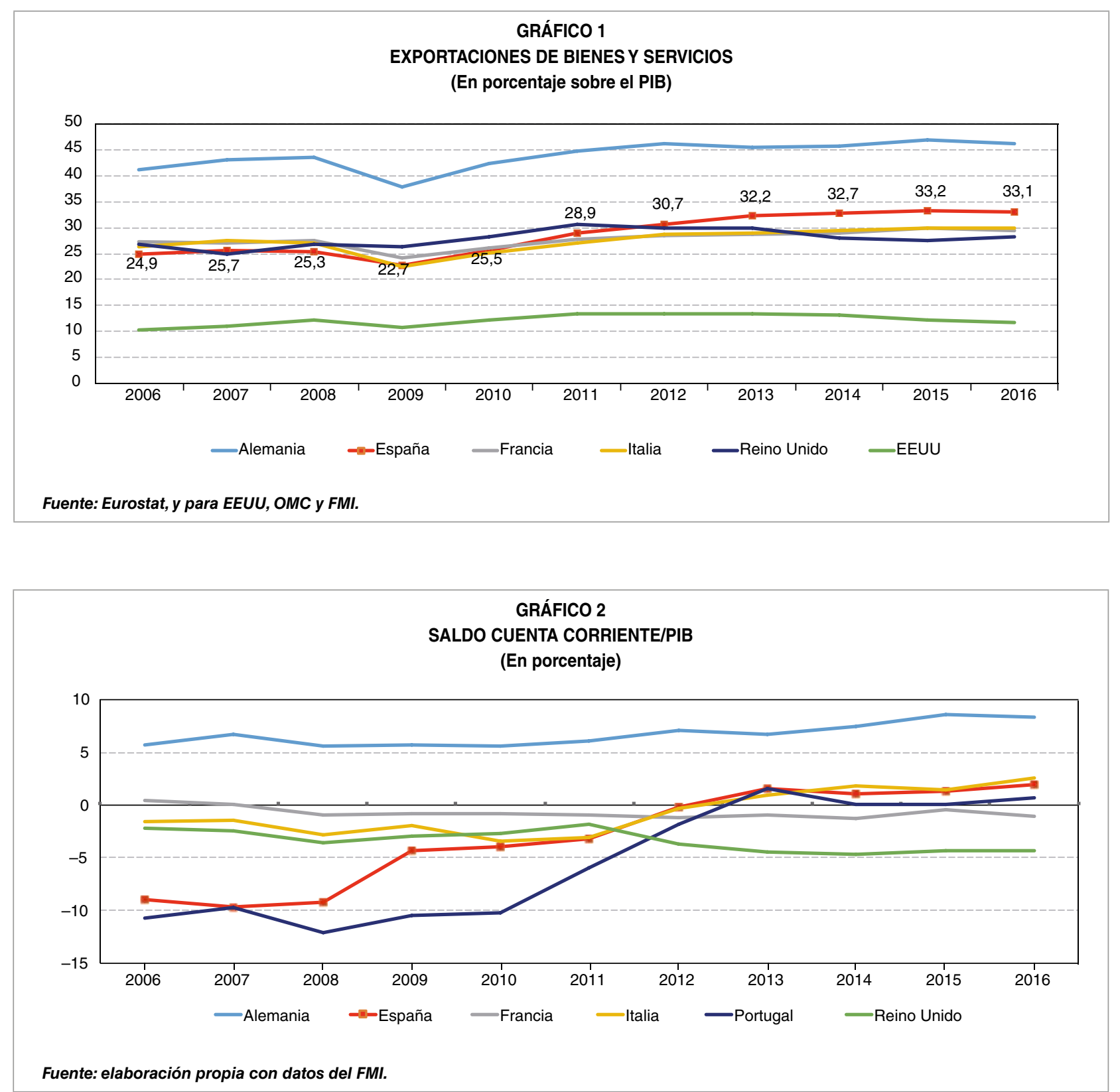

1. El nivel de exportaciones de bienes y servicios es un tercio mayor.

2. Nuestra economía es ahora mucho más abierta, pues el porcentaje de exportaciones de bienes y servicios sobre el PIB ha pasado del 25 por 100 en 2008 al 33 por 100 en 2016, superando a países como Francia, Italia y Reino Unido.

3. El déficit comercial se ha reducido en 81.000 millones de euros, fundamentalmente por el aumento de las exportaciones totales $y$, en una medida mucho menor, por el abaratamiento de la factura energética.

4. A partir de julio de 2012, el saldo de la cuenta corriente de la Balanza de Pagos empezó a registrar saldo positivo. De hecho, España ha tenido cuatro años de superávit corriente (desde 2013) y cinco años de capacidad de financiación $\triangle$ 
al exterior (desde 2012), y está previsto que siga esta tendencia en 2017 y 2018.

5. Esta buena evolución del saldo de la cuenta corriente ha permitido corregir en 12 puntos el desequilibrio exterior (España tenía el segundo déficit corriente más alto del mundo en el año 2007 en valores absolutos, casi un 10 por 100 en términos de PIB).

6. Hemos pasado de tener una necesidad de financiación del 8,8 por 100 del PIB a conseguir una capacidad de financiación del 2,1 por 100.

En el momento actual, la exportación española está alcanzando cifras nunca vistas y nuestro déficit comercial registró el año pasado el segundo mejor saldo de los últimos veinte años. En España, hoy en día, 4,6 millones de puestos de trabajo dependen de la actividad exterior de las empresas.

\section{3. ¿Por qué una estrategia de internacionalización?}

Ante esta situación, habrá quién se pregunte por qué ha sido precisamente ahora cuando el Gobierno ha decidido elaborar una estrategia de internacionalización a medio plazo. La respuesta es muy clara: porque nuestro país no se puede arriesgar a que el buen comportamiento del sector exterior se limite a los momentos de crisis económica, de debilidad de la demanda interna, como ha sucedido en otras ocasiones.

Tradicionalmente, nuestro sector exterior ha sido el motor de salida de las diferentes crisis económicas por las que hemos pasado en las últimas décadas, contribuyendo de forma positiva al crecimiento del PIB y compensando así la caída de la demanda interna. Pero, una vez superada la crisis, las empresas siempre han dejado de lado, en mayor o menor medida, su actividad exportadora.

El Gobierno no quiere que este repliegue de las empresas al mercado nacional vuelva a suceder ahora, una vez que lo peor de la crisis económica ha pasado. De ahí que haya decidido elaborar esta estrategia de internacionalización en este momento, ya que aspira a que el sector exterior se convierta en un componente estructural $-\mathrm{y}$, por tanto, permanente- de nuestro modelo de crecimiento, porque así es como se podrán conseguir mayores ritmos de crecimiento y de creación de empleo. Actualmente, uno de cada cuatro puestos de trabajo en nuestro país depende de la actividad internacional de las empresas, y lo que se busca con esta estrategia es ir dando los pasos para que esta cifra no solo se mantenga, sino que aumente.

\section{Principios rectores}

La Estrategia de Internacionalización 20172027 se rige por dos principios fundamentales: coherencia y coordinación de la acción de las Administraciones Públicas en materia de internacionalización, y complementariedad con la actuación del sector privado, identificando en qué aspectos el potencial del sector privado debe ser complementado por la acción del Gobierno.

Dado que los recursos públicos son limitados, es fundamental maximizar la eficiencia de la actuación pública. Para ello debemos dar prioridad, desde el punto de vista geográfico y sectorial, a aquellos mercados más atractivos para el conjunto de la oferta española, pues no se puede justificar la actuación pública en apoyo de las empresas en todos los mercados. $\square$ 


\section{Análisis de la situación actual de nuestro sector exterior: fortalezas, debilidades y retos}

La estrategia parte de un análisis de la situación actual de nuestro comercio e inversiones exteriores para conocer sus fortalezas y debilidades.

\subsection{Comercio exterior}

Destaca el crecimiento sostenido de nuestras exportaciones en un contexto de ralentización del comercio mundial, lo que ha permitido que España, durante los últimos ocho años, haya conseguido mejorar su participación en las exportaciones mundiales.

En 2016, España subió dos puestos y se situó en el decimosexto lugar en el ranking mundial de exportaciones de mercancías, con una cuota de mercado del 1,8 por 100 sobre el total mundial (frente al puesto vigesimoprimero, con un 1,6 por 100 en 2012), y en el undécimo puesto en el caso de las exportaciones de servicios, con una cuota del 2,7 por 100 (2,5 por
100 en 2015). Es todavía más destacable que, mientras que el resto de los principales países europeos de nuestro entorno han visto retroceder su cuota de mercado mundial desde 2006, España haya logrado prácticamente mantener su participación en las exportaciones mundiales en un periodo caracterizado por una fuerte irrupción de las economías emergentes en el comercio mundial. Esto supone, por tanto, un hecho diferencial del sector exterior español.

Los factores que explican este buen comportamiento de nuestro comercio exterior son varios. Entre ellos destaca la mejora de la competitividad de nuestras exportaciones, la mejor inserción de España en las cadenas globales de valor y el aumento del número de empresas exportadoras de todos los tamaños, en especial de las de menor volumen de exportación. En el año 2016 se contabilizaron 148.794 empresas exportadoras (un 21 por 100 más que en 2011) y 49.792 que exportaron de forma regular (un 34 por 100 más que en 2011).

No obstante, el proceso de internacionalización de nuestra economía presenta ciertas debilidades y tiene ante sí, por tanto, una serie de retos. Entre las debilidades destacan:

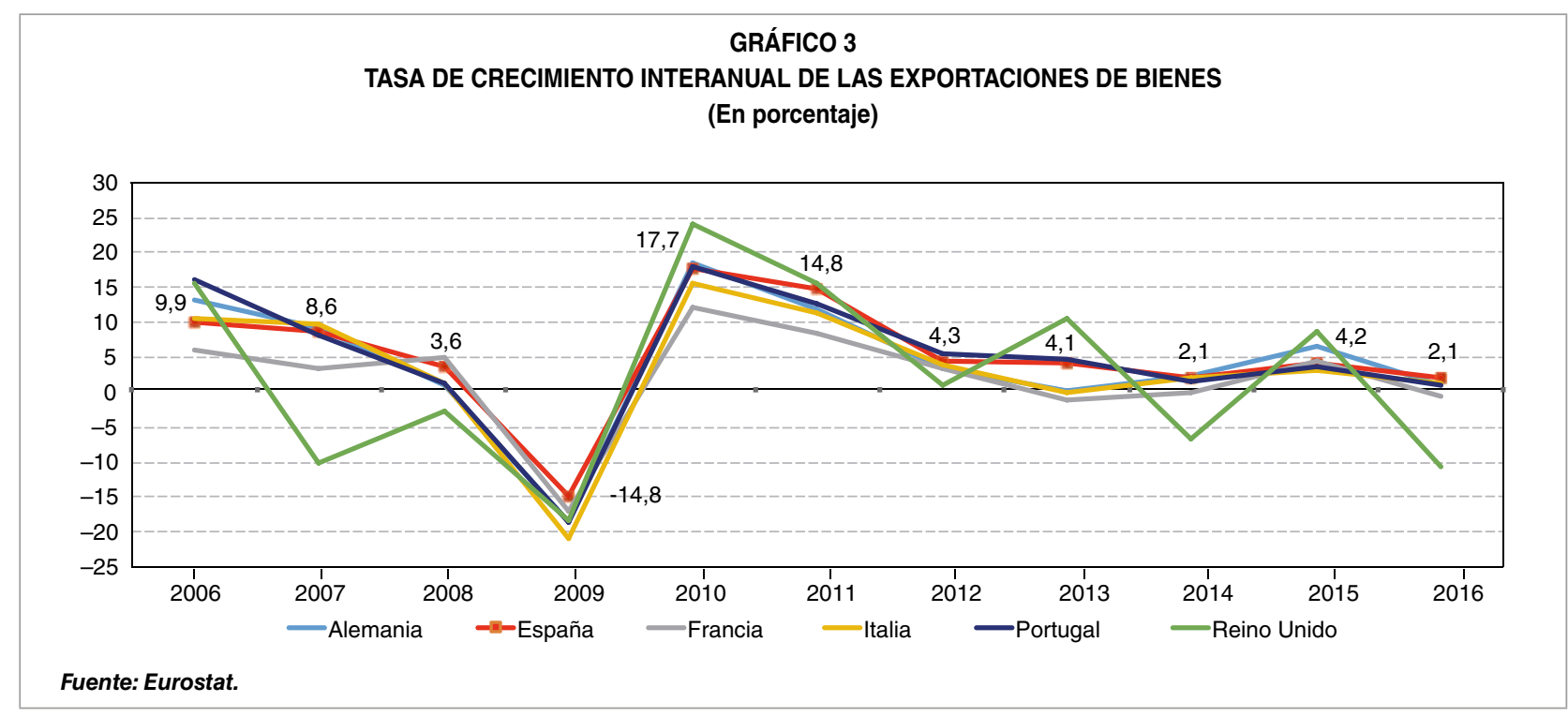


1. La concentración de la exportación en un número reducido de empresas: en 2016 las empresas que exportaron más de 250 millones de euros anuales suponen el 0,1 por 100 de las empresas exportadoras y el 40 por 100 de la exportación.

2. La escasa propensión a exportar de las pymes: según los últimos datos disponibles, en el año 2014 solamente un 3 por 100 de las empresas registradas en el DIRCE (Directorio Central de Empresas), con plantilla inferior a 200 trabajadores, desarrolló actividades de exportación.

3. La aún insuficiente diversificación de los mercados de destino de nuestra exportación: a pesar de la progresiva diversificación de nuestras exportaciones, el 66 por 100 de ellas tuvieron como destino países de la UE-28 en al año 2016.

4. La insuficiente presencia en los mercados de Asia, particularmente en China y Japón, y en Estados Unidos, que representan conjuntamente el 30 por 100 de las exportaciones mundiales y en los que España se encuentra proporcionalmente mucho más lejos que nuestros competidores. Sin crecimiento en estos mercados resultará difícil incrementar nuestra cuota en los mercados mundiales.

5. El todavía insuficiente nivel medio del contenido tecnológico de las exportaciones: si bien más de la mitad de la exportación española de bienes se encuadra, por su contenido tecnológico, en los segmentos alto y medio-alto, se observa en los últimos años una estabilización de estos grupos en el total de la exportación (55,45 por 100 en 2006; 55,3 por 100 en 2016).

6. La limitada propensión a exportar de algunas comunidades autónomas: seis de ellas (Cataluña, Madrid, Andalucía, Comunidad Valenciana, País Vasco y Galicia, por este orden) son responsables de las tres cuartas partes de nuestras exportaciones (74,4 por 100). Atendiendo a la propensión a exportar (exportaciones de manufacturas en porcentaje de la producción de la industria manufacturera), según los datos de 2015, Madrid, Cataluña, Illes Balears, Galicia y Navarra presentan una propensión a exportar por encima de la media nacional $(60,5$ por 100$)$.

\subsection{Inversiones exteriores}

Con respecto a la inversión extranjera en nuestro país, tras unos años de comportamiento errático debido a la desfavorable evolución económica nacional e internacional, la inversión extranjera directa (IED) en España se está recuperando de forma sostenida. La atracción de inversiones extranjeras tiene gran interés, ya que no solo permite incrementar el stock de inversión en el país, sino que además tiene efectos muy positivos en la generación y mantenimiento de puestos de trabajo y en la actividad de las empresas, así como en el desarrollo económico a través de la difusión del conocimiento y la tecnología, el fomento de la competencia, el mayor desarrollo del capital humano nacional y, en definitiva, el impulso de la competitividad.

La inversión extranjera neta sin entidades de tenencia de valores extranjeros se incrementó en 2013 en un 41 por 100, se mantuvo en 2014 y se incrementó otro 44 por 100 en 2015, alcanzando la cifra de 17.904 millones de euros y manteniéndose con una pequeña disminución del 2 por 100 hasta los 17.535 millones de $\triangleright$ 
euros en 2016. Los países con mayor posición inversora en España son Estados Unidos, Reino Unido, Italia y Francia. Según los datos de inversión extranjera directa en stock de la UNCTAD (Conferencia de las Naciones Unidas sobre Comercio y Desarrollo, por sus siglas en inglés), España es el 13. mayor receptor a nivel mundial de IED en 2015, con un stock que supera los 533.300 millones de dólares (cifra que representa el 44,4 por 100 de nuestro PIB). En nuestro país hay establecidas unas 12.400 empresas de capital extranjero, que emplean a 1.183.000 trabajadores.

La inversión española en el exterior también ha repuntado en los últimos años, especialmente en 2015, si bien en 2016 ha disminuido, aunque todavía presenta valores netos por encima de años anteriores. La evolución de los flujos de inversión neta española en el exterior descendió un 29 por 100 en 2016 respecto a 2015, hasta los 19.733 millones de euros, encontrándose en valores muy superiores al año 2014 (es preciso tener en cuenta que 2015 fue un año excepcional con grandes operaciones puntuales).

La posición inversora total española en el exterior (stock), incluyendo las ETVE, tuvo un comportamiento positivo, aumentando desde los 459.536 millones de euros en 2014 hasta los 472.760 millones de euros en 2015. De esta forma, el stock español en el exterior superó al extranjero en España en 85.445 millones de euros en 2015. Por países, Reino Unido es el primer destino de nuestras inversiones exteriores. Le siguen Estados Unidos, Brasil y México.

\subsection{Retos de nuestro sector exterior}

Teniendo en cuenta las fortalezas y debilidades de nuestro sector exterior, los retos a los que se enfrenta son, por tanto, los siguientes:

1. Incrementar y consolidar la base de empresas que exportan regularmente, que no se repliegan hacia el mercado interno una vez superada la crisis, para asegurar así que la aportación del sector exterior al crecimiento sea de carácter estructural.

2. Centrar la estrategia alrededor de las pymes y propiciar la adopción de medidas que favorezcan el aumento del tamaño de la empresa para su internacionalización, y también aquellas que permitan o faciliten la integración entre empresas, pues la gran atomización del tejido empresarial español es una de las principales razones por las que el número de empresas que se internacionalizan es pequeño en comparación con otros países de nuestro entorno. La pequeña dimensión de la pyme se traduce en menor productividad, falta de fuerza para negociar con proveedores y escasez de recursos que puede dedicar a internacionalización e innovación.

3. El desarrollo tecnológico y la innovación también deben constituir una prioridad. Las empresas españolas deben incrementar su competitividad a través de la generación e incorporación de conocimientos, tecnologías e innovaciones destinadas a la mejora de los procesos y la creación de productos y servicios tecnológicamente avanzados y de mayor valor añadido. Ello permitirá incrementar el peso de las exportaciones de productos de alto nivel tecnológico y valor añadido, permitiendo también un incremento de la competitividad de nuestro sector exterior. 
4. Durante los próximos años ningún sector podrá quedar al margen de la transformación digital, que modificará la forma de hacer negocios, los productos y servicios disponibles, los canales de venta o los mecanismos de relación con el consumidor. Los avances en materia de digitalización tienen un efecto transversal y multiplicador sobre un número importante de actividades claves en la economía, y la Administración debe poder acompañar a las empresas en el proceso de transformación digital de su modelo de negocio, trabajando también para que los acuerdos comerciales con países terceros recojan disposiciones que faciliten el desarrollo del comercio electrónico.

5. Insertar la exportación española de forma estratégica en las cadenas globales de valor, pues éstas generan cerca del 80 por 100 del comercio global a través del comercio transfronterizo de inputs y outputs entre su red de filiales, socios y proveedores. Fomentan la transferencia tecnológica, la productividad y la competitividad industrial y promueven la aparición de proveedores de bienes intermedios. Constituyen, por tanto, una vía para conseguir una mayor especialización y valor añadido de nuestras exportaciones.

6. Continuar fomentando la capacitación empresarial y el acceso de nuestras pymes a información de calidad sobre mercados y sectores que sea útil en su proceso de toma de decisiones, así como sobre los distintos instrumentos de internacionalización. Igualmente, es clave la formación de su capital humano.
7. Es importante que seamos capaces de atraer talento, pues la atracción de profesionales altamente cualificados y emprendedores mejoran la competitividad de las empresas.

8. Uno de los principales cuellos de botella es la dificultad de nuestras pymes para acceder a mejor financiación (obtener garantías y avales con los que adquirir financiación bancaria, así como acceder a fuentes de financiación alternativas a la bancaria), tanto para financiar la actividad propia como para ofrecer a sus compradores.

9. Contar con mercados cada vez más abiertos en los que se respeten las reglas de las que nos hemos dotado. Para ello, la política comercial común debe continuar desarrollándose a través de la firma de acuerdos comerciales con terceros países que reflejen mejor los intereses españoles. Estos acuerdos son el mejor instrumento para abrir mercados y para eliminar las barreras arancelarias y no arancelarias que se detecten.

Como resultado del análisis anterior, se puede construir una matriz resumen de las Debilidades, Amenazas, Fortalezas y Oportunidades (DAFO) que tiene España a la hora de definir la estrategia de internacionalización y que vemos reflejada en el Cuadro 1.

\section{Objetivos de la estrategia}

La Estrategia de Internacionalización de la Economía Española 2017-2027 y sus planes de acción bienales constituyen la pieza fundamental para alcanzar los retos existentes, siendo su objetivo general afianzar la contribución positiva y permanente del sector exterior a $D$ 


\section{LA ESTRATEGIA DE INTERNACIONALIZACIÓN DE LA ECONOMÍA ESPAÑOLA 2017-2027}

CUADRO 1

ANÁLISIS DAFO

\begin{tabular}{|c|c|c|}
\hline & Fortalezas & Debilidades \\
\hline \multirow{18}{*}{$\begin{array}{l}\text { Análisis } \\
\text { interno }\end{array}$} & Aumento de la propensión a exportar bienes y servicios & Concentración exportación en número reducido empresas \\
\hline & \multirow{3}{*}{ Incremento de la cuota de exportación mundial } & Importante desequilibrio comercial con algunos países \\
\hline & & Escasa propensión a exportar de la pyme \\
\hline & & $\begin{array}{l}\text { Importante peso del mercado británico en nuestra exporta- } \\
\text { ción: riesgo y consecuencias del brexit }\end{array}$ \\
\hline & $\begin{array}{l}\text { Crecimiento de las exportaciones a mercados no } \\
\text { comunitarios }\end{array}$ & Insuficiente diversificación geográfica de las exportaciones \\
\hline & $\begin{array}{l}\text { Incremento de la base exportadora y de los márgenes } \\
\text { extensivo e intensivo }\end{array}$ & Limitada propensión a exportar de determinadas CCAA \\
\hline & $\begin{array}{l}\text { Aumento de exportaciones de contenido tecnológico medio } \\
\text { y medio-alto }\end{array}$ & $\begin{array}{l}\text { Todavía insuficiente contenido tecnológico de las } \\
\text { exportaciones } \\
\text { Insuficiente componente marquista de las exportaciones }\end{array}$ \\
\hline & $\begin{array}{l}\text { Crecimiento de las exportaciones de servicios turísticos y } \\
\text { no turísticos }\end{array}$ & \\
\hline & Ganancias de competitividad precio y no precio & $\begin{array}{l}\text { Elevada propensión a importar cuando la economía se } \\
\text { recupera }\end{array}$ \\
\hline & Favorable inserción en las cadenas de valor global & $\begin{array}{l}\text { Reducción de la participación Forward en las cadenas de } \\
\text { valor global }\end{array}$ \\
\hline & Consolidación de los superávits por cuenta corriente & Riesgo de subida en el precio del petróleo y tipos de interés \\
\hline & $\begin{array}{l}\text { Esfuerzo para incorporar la agenda digital en los modelos } \\
\text { de negocio }\end{array}$ & Bajo grado de digitalización de las empresas \\
\hline & $\begin{array}{l}\text { Creciente cultura de internacionalización entre las em- } \\
\text { presas. Cambio de mentalidad sobre el papel clave de la } \\
\text { internacionalización }\end{array}$ & $\begin{array}{l}\text { Insuficiente formación en internacionalización en los } \\
\text { cuadros directivos de las empresas, particularmente } \\
\text { pymes }\end{array}$ \\
\hline & Recuperación de forma sostenida de la inversión extranjera & $\begin{array}{l}\text { Posición neta de inversión internacional negativa, de las } \\
\text { más altas del mundo en relación al PIB. Necesidad de } \\
\text { potenciar la atracción de inversión extranjera }\end{array}$ \\
\hline & $\begin{array}{l}\text { Fuerte repunte de la inversión exterior desde } 2015 \text { con } \\
\text { valores netos por encima de años anteriores }\end{array}$ & $\begin{array}{l}\text { Necesidad de seguir aplicando políticas de apoyo a la } \\
\text { inversión exterior }\end{array}$ \\
\hline & Existencia de un amplio número de instrumentos y organis- & $\begin{array}{l}\text { Necesidad de mayor coherencia y colaboración entre los } \\
\text { mismos }\end{array}$ \\
\hline & mos de apoyo a la internacionalización & $\begin{array}{l}\text { Dificultad de acceder a la información relativa a dichos } \\
\text { instrumentos }\end{array}$ \\
\hline & Oportunidades & Amenazas \\
\hline \multirow{3}{*}{$\begin{array}{l}\text { Análisis } \\
\text { externo }\end{array}$} & $\begin{array}{l}\text { Previsión de recuperación del crecimiento del comercio } \\
\text { mundial en } 2017(2,4 \%) \text { y en } 2018(2,1 \%-4,0 \%)\end{array}$ & $\begin{array}{l}\text { Creciente competencia de economías emergentes en el } \\
\text { comercio mundial de bienes y servicios }\end{array}$ \\
\hline & $\begin{array}{l}\text { Firma de acuerdos comerciales de la UE con terceros paí- } \\
\text { ses que reflejen los intereses de nuestras empresas }\end{array}$ & $\begin{array}{l}\text { Incremento de las tentaciones proteccionistas } \\
\text { Insuficiente comunicación sobre las oportunidades que } \\
\text { ofrecen los acuerdos comerciales a las empresas }\end{array}$ \\
\hline & Previsión de crecimiento de la inversión mundial & $\begin{array}{l}\text { Creciente competencia de economías emergentes y de los } \\
\text { países de nuestro entorno en captación de flujos de inversión }\end{array}$ \\
\hline
\end{tabular}

nuestro crecimiento económico, consiguiendo así una mayor creación de empleo.

De aquí a diez años, a través de la Estrategia de Internacionalización se busca alcanzar, en concreto:
1. Un valor de exportaciones nominales de bienes de 400.000 millones de euros en 2027.

2. Conseguir que 60.000 empresas exporten de forma regular. 
3. Conseguir que en el periodo 2017-2027, las empresas españolas resulten adjudicatarias de 650.000 millones de euros de licitaciones internacionales.

4. Alcanzar un stock de inversiones directas extranjeras de 500.000 millones de euros en 2027.

\section{Ejes de la estrategia}

Para alcanzar estos retos, la estrategia se articula en torno a seis ejes principales, en los que se encuadran una serie de medidas o actuaciones concretas que conforman los distintos planes de actuación bienales.

\section{Primer eje. Ofrecer un apoyo a la} internacionalización cada vez más adaptado a las necesidades y al perfil de nuestras empresas

Para conseguir incrementar la base de empresas que exportan de forma regular es necesario un mejor conocimiento de las empresas, sobre todo de aquéllas que no mantienen su actividad internacional de forma constante. Se hace necesario, por tanto, diagnosticar mejor sus necesidades, realizar una mayor segmentación del tejido empresarial — para conseguir una mayor adaptación de los programas y los servicios de promoción de exportaciones e inversiones-y elaborar programas que les permitan abordar la actividad internacional de forma más regular.

Segundo eje. Incorporar la innovación, la tecnología, la marca y la digitalización a la internacionalización

El nivel tecnológico tiene efecto directo en la competitividad del país, por lo que es crucial que instituciones, empresas y entidades financieras trabajemos conjuntamente para apoyar proyectos innovadores que contribuyan a crear empleo de calidad y a renovar estructuras productivas para dar paso a otras más modernas e innovadoras. La marca, por su parte, responde a la necesidad de la empresa por diferenciar sus productos. Hoy en día, para afrontar con éxito la internacionalización, es también imprescindible que las empresas se incorporen al mundo digital, que sin duda redundará en mayores beneficios y más presencia internacional de nuestras empresas.

Tercer eje. Desarrollar el capital humano para la internacionalización

El progreso de la industria 4.0 y la consolidación de las nuevas tecnologías en las formas de establecer y desarrollar todos los procesos del negocio internacional afectarán también a la gestión del talento en las organizaciones. Es necesario, por tanto, que desde la Administración sigamos desarrollando medidas que permitan la captación de talento y estimulen la cultura de la internacionalización y la formación de capital humano especializado.

\section{Cuarto eje. Aprovechar mejor las oportunidades de negocio derivadas de la política comercial común y de las instituciones financieras y organismos multilaterales}

Para que las empresas puedan contar con mercados terceros cada vez más abiertos, la política comercial común debe continuar desarrollándose a través de la firma de acuerdos comerciales con terceros países y de la detección y eliminación de barreras al comercio y a la inversión. Desde la Secretaría de Estado de Comercio se seguirá trabajando para defender los intereses y necesidades españoles, tratando de aumentar nuestra capacidad $D$ 
de influencia en la UE y en la Organización Mundial de Comercio (OMC). Asimismo, también se seguirá apoyando a nuestras empresas para aprovechar al máximo el potencial de los proyectos con financiación multilateral.

\section{Quinto eje. Potenciar la captación y} consolidación de la inversión extranjera de alto valor añadido

En un contexto de creciente competencia entre los países de nuestro entorno por la captación de inversiones, es necesario continuar trabajando para atraer inversión extranjera a nuestro país. Para ello, se trabajará en un programa de acciones de promoción en países-objetivo encaminadas a posicionar España como plataforma de inversiones ante entidades financieras y fondos, ante empresas facilitadoras/prescriptoras y ante inversores industriales, tecnológicos y de servicios.

\section{Sexto eje. Reforzar la coordinación y} complementariedad de acciones entre todos los actores relevantes en materia de internacionalización

Desde esta Secretaría de Estado de Comercio se centrarán los esfuerzos para que todos los ministerios y organismos con actuaciones en materia de fomento de la internacionalización trabajen de forma coordinada y eficiente, junto al sector privado, con el fin de ofrecer el mejor servicio posible a las empresas españolas.

Cada uno de estos ejes incorpora una serie de medidas concretas, que conforman los planes de acción bienales que irán desarrollando la estrategia a diez años. El primero de ellos, el
Plan de Acción 2017-2018, está incluido en el documento aprobado por el Consejo de Ministros el pasado septiembre.

La estrategia se completa con siete anexos, con información adicional y detallada sobre los instrumentos de apoyo a la internacionalización en el ámbito del Ministerio de Economía, Industria y Competitividad, sobre la política comercial común de la UE y la OMC, sobre la participación española en instituciones multilaterales, sobre los indicadores de seguimiento y sobre las actividades de otros ministerios en materia de internacionalización.

\section{Conclusión}

La Estrategia de Internacionalización de 2017-2027 muestra el grado de ambición del Gobierno y su compromiso con el impulso de la internacionalización de nuestra economía. Como se ha expuesto, este documento fue aprobado por el Consejo de Ministros y constituye, por tanto, una política del Gobierno. Adicionalmente, el Gobierno aspira a contar con el apoyo de los diferentes partidos políticos en el seno del Congreso y del Senado para poder conseguir algo que el sector privado viene reclamando desde hace tiempo: que el fomento de la internacionalización se convierta en una política de Estado.

La Estrategia de Internacionalización de la Economía Española 2017-2027 se puede descargar en el siguiente enlace: $h t t p: / / w w w . m i n e-$ co.gob.es/stfls/mineco/comercio/pdf/170913_ Estrategia_Internacionalizacion_2017.pdf 


\section{Información Comercial Española \\ Revista de Economía}

6 números anuales

Artículos originales sobre un amplio espectro de temas tratados desde

una óptica económica, con especial referencia a sus aspectos internacionales

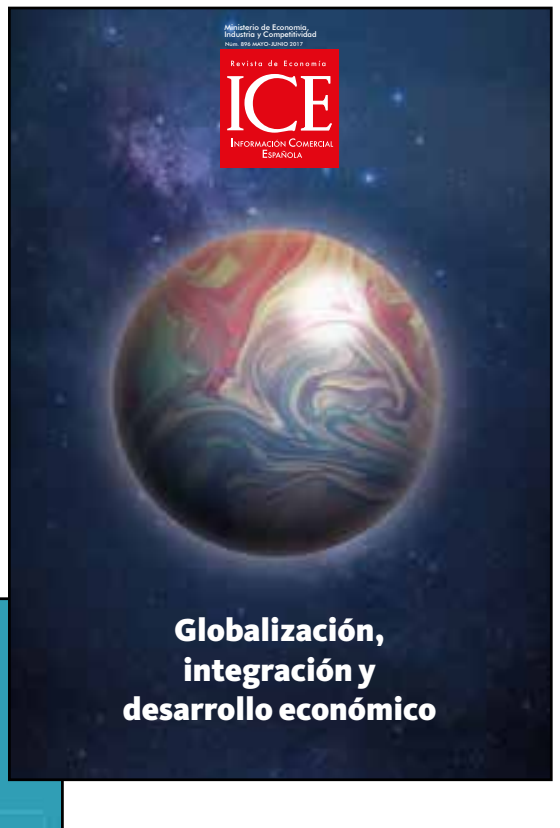

de Información Comercial Española

12 números anuales

Artículos y documentos sobre economía española, comunitaria e internacional, con especial énfasis en temas sectoriales $y$ de comercio exterior

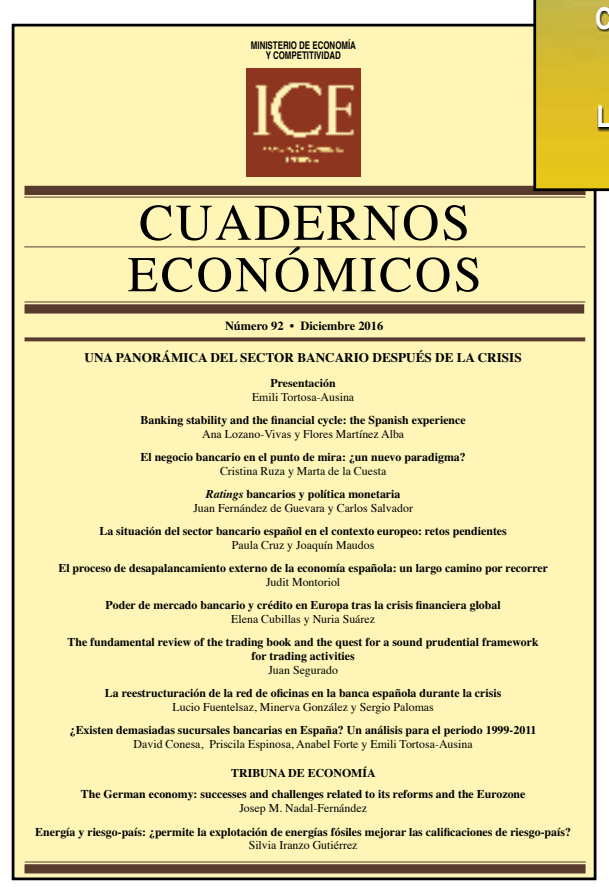

Cuadernos Económicos de ICE

2 números anuales

Artículos de economía teórica y aplicada y métodos cuantitativos, que contribuyen a la difusión y desarrollo de la investigación 